\title{
Evaluation of trabecular bone microstructure of mandibular condyle in edentulous, unilateral edentulous and fully dentate patients using cone-beam computed tomography
}

\author{
A. Koç'1, i. Kavut², M. Uğur² \\ 'Department of Oral and Maxillofacial Radiology, Van Yüzüncü Yıl University Faculty of Dentistry, Van, Turkey \\ 2Department of Prosthetic Dentistry, Van Yüzüncü Yıl University Faculty of Dentistry, Van, Turkey
}

[Received: 10 October 2019; Accepted: 22 November 2019]

Background: The aim of this study was to compare the trabecular bone microstructure of the mandibular condyle in edentulous, unilateral edentulous (Kennedy Class II), and fully dentate patients.

Materials and methods: The study used the cone-beam computed tomography (CBCT) images of 17 fully dentate (34 condyles), 16 edentulous (32 condyles), and 17 unilateral edentulous patients (34 condyles) aged 19 to 80 years. The trabecular bone microstructure of the mandibular condyle was evaluated on 8 consecutive cross-sectional images of these patients. In the microstructure analysis, structural model index (SMI), ellipsoid factor (EF), bone volume fraction (BV/TV), trabecular thickness (Tb.Th) and trabecular seperation (Tb.Sp) were measured.

Results: There was no significant difference between the mean SMI, BV/TV, EF and Th. Th microstructure values of each group $(p=0.243, p=0.095, p=0.962$, $p=0.095$, respectively). However, there was significant difference in terms of mean Tb.Sp between the groups $(p=0.021)$. The trabecular structure in all three groups was more rod-shaped. No correlation was found between age factor and microstructure values.

Conclusions: Considering the in vivo microstructure analysis of CBCT images, it can be said that teeth loss does not have a significant effect on the microstructure parameters excluding Tb.Sp of mandible condyles and does not affect mandibular condyle trabecular endurance. (Folia Morphologica 2020; 79, 4: 829-834)

Key words: mandibular condyle, cone-beam computed tomography, quantitative evaluation, bone tissue

\section{INTRODUCTION}

The facial profile, as well as environmental and genetic factors, may affect the masticatory system of individuals and subsequently affect bone morphology in the mandible $[3,21]$. The temporomandibular joint (TMJ) may be exposed to degenerative changes when there are changes in masticatory functions or when force is applied powerful enough to disrupt the joint structure [22]. Furthermore, sudden changes in masticatory functions may deteriorate the ongoing delicate balance between dental occlusion, masticatory muscles and TMJ structure, and the deteriorated balance may concentrate on a different system [19, 23]. Loss of occlusal support deteriorating the balance in the masticatory system may be one of the factors that accelerate TMJ deterioration. Premature contacts

Address for correspondence: Dr. A. Koç, Van Yüzüncü Yıl University, Faculty of Dentistry, Department of Oral and Maxillofacial Radiology, 65080, Van, Turkey, tel: +90 43 22251744, fax: +90 43 22251747, e-mail: alttnkc@gmail.com 
have been found to be present in patients with TMJ disorder and this abnormal relationship has been observed to be more frequent in these patients than people with normal joints. This imbalance can lead to degenerative changes due to increased stress on the masticatory muscles and excessive pressure on the joint surface [26]. In the literature, techniques such as electromyography and kinesiography have been used to analyse the relationship between dental malocclusion and TMJ disorder. However, no significant correlation has been found between dental malocclusion and TMJ disorder with the above-mentioned techniques [20].

Edentation, teeth movements, and parafunctional habits such as bruxism are effective in the formation of occlusal force. Parameters such as dentition status, occlusal force, internal structure and morphology of TMJ are known to be correlated with each other. Considering the correlation between these parameters, it is possible that the trabecular structures of the mandibular condyle of the edentulous and fully dentate patients are of different types [5]. In other words, properties such as density, thickness, and seperation of the trabecular structure may change due to the above-mentioned external factors [14]. For instance, it has been reported that the density and bone volume fraction (BV/TV) of mandibular condyle trabeculae are lower in edentulous patients than in fully dentate patients [13]. Similarly, in a different study [11], patients with high bone densities were found to have a plate-like trabecular structure whereas patients with low bone densities had a rodlike trabecular structure considering the relationship between mandibular condyle trabecular structure and density of related bone of the fully dentate patients.

Different imaging techniques such as plain radiographs, computed tomography (CT), magnetic resonance imaging (MRI) and cone-beam CT (CBCT) have been used to examine the TMJ $[20,23,26]$. A three-dimensional (3D) image of the mandibular condyle can be obtained, analysis of surrounding tissues and morphological changes in bones can be examined, and the cavity and dynamic functioning of the TMJ can be analysed using CT, MRI and CBCT techniques. There is an increasing demand for $\mathrm{CBCT}$ devices because it has voxel dimensions of up to $0.075 \mathrm{~mm}$, has exact measurement capability, and requires low dose and cost compared to multi-slice CT (MSCT) devices $[1,16,17]$. Although Hounsfield unit values could not be determined exactly with
$\mathrm{CBCT}$, bone microstructure values were measured using CBCT in some studies $[18,24]$. Microstructure evaluation values close to micro-CT devices, which are accepted as the gold standard for the evaluation of bone microstructure, have been reported to be obtained with CBCT with the help of images taken at low voxel dimensions [24].

To the best of our knowledge, there is no study in the literature that compares the mandibular condyle trabecular structure parameters of edentulous, unilateral edentulous, and fully dentate patients. The aim of this study was to compare the bone volume fraction (BV/TV), structural model index (SMI), ellipsoid factor (EF), trabecular thickness (Tb.Th) and trabecular seperation (Tb.Sp) parameters of the groups and to determine the effect of loss of occlusion on the trabecular bone structure of the mandibular condyle.

\section{MATERIALS AND METHODS}

A total of 50 patients ( 25 female and 25 male) aged 19-80 years were classified according to their edentation status. Of these patients, 17 were fully dentate with 34 condyles (mean age 40.7), 16 were edentulous with 32 condyles (mean age 52.3), and 17 were unilateral edentulous with 34 condyles (mean age 56.9). Trabecular bone microstructures of mandible condyle were analysed on their cross-sectional images. Patients with systemic diseases that can affect the bone metabolism and microstructure, deformity, fracture, or tumoral lesion in the mandibular condyle region and those having cross-sectional images with poor quality were excluded from the study. KaVo 3D eXam (Biberach, Germany) tomography device was used to obtain the images. Scanning parameters were selected as follows: $20.27 \mathrm{~mA}, 14.7 \mathrm{~s}, 120 \mathrm{kVp}$, $16 \times 6 \mathrm{~cm}$ field of view, and $0.2 \mathrm{~mm}$ isotropic voxel. The gonion of the patients was fixed with a plastic holder to prevent movement artifact during screening. This study was conducted following the receipt of approval from University Ethical Committee (Approval no: Van YYU-2018/04/04-05).

Cone-beam CT scanning data was converted to digital imaging and communications in medicine (DICOM) format. Cross-sectional images of the patients' mandibular condyle region were imported into ImageJ software (U.S. National Institutes of Health, Bethesda, Maryland, USA). Eight consecutive sections without intersection gaps between each other were stacked into ImageJ software. In microstructure analysis, $2 \mathrm{~mm}$ apical of the cortical bone at the head of 

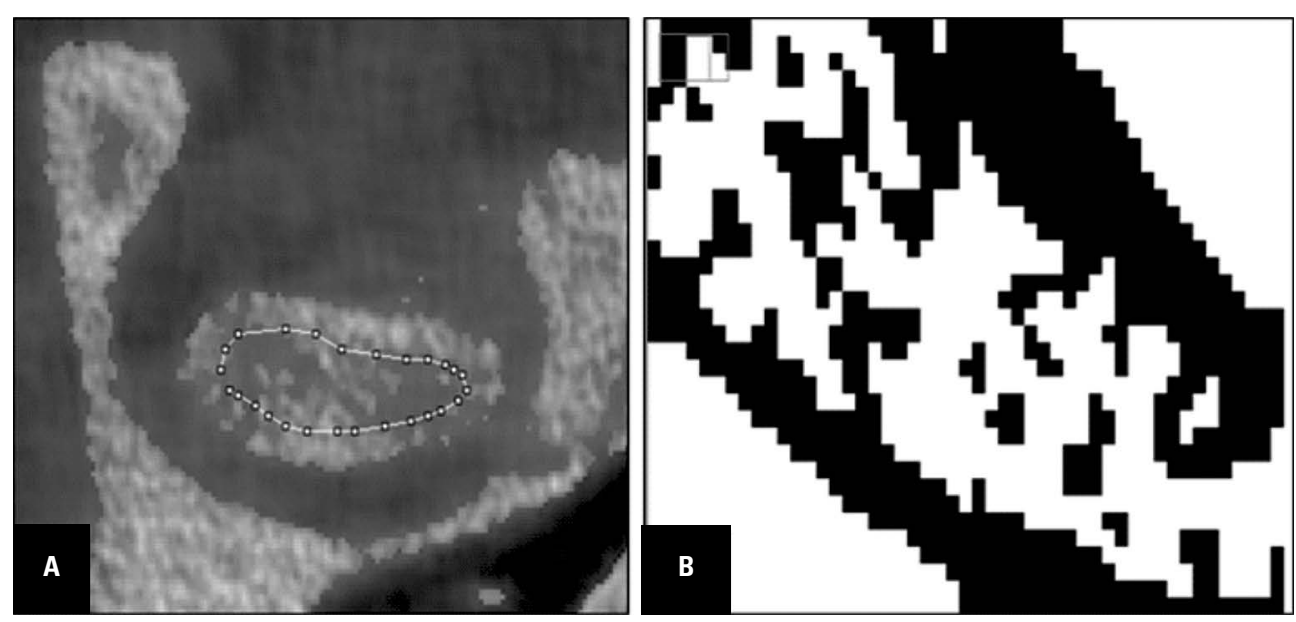

Figure 1. Selection of region of interest in axial sectional image (A), and conversion of sectional image into binary image format to make suitable for microstructure analysis (B).

the condyle and trabecular bone area within $2 \mathrm{~mm}$ of the cortical bone of the condyle in each axial sectional image were included while selecting the region of interest (ROI). The ROI was duplicated to be measured and then the images were converted to binary image form to make them suitable for microstructure evaluation (Fig. 1). Then, SMI, BV/TV, $\mathrm{EF}, \mathrm{Tb}$.Th and Tb.Sp values were measured by BoneJ plugin in the software. Measurements were made by an oral and maxillofacial radiologist who have been analysing CBCT images for 3 years and performing microstructure analysis with related software for 2 years.

\section{Statistical analysis}

Statistical analysis of the data obtained was performed using SPSS version 20.0 software (IBM SPSS Statistics 20.0; IBM Co., Armonk, NY, USA). The relationship between loss of occlusion and SMI, BV/TV, $\mathrm{EF}, \mathrm{Tb}$.Th and Tb.Sp values was analysed by one-way ANOVA test. Mann-Whitney $U$ test was used to compare the trabecular microstructure parameters of condyles belonging to edentulous and dentate sides in unilateral edentulous patients. Intra-class correlation coefficient (ICC) was calculated to measure intra-rater reliability. Randomly selected trabecular bone microstructures of 34 condyle heads were analysed by an observer twice in 1-month intervals.

\section{RESULTS}

Descriptive statistics of the parameters are presented in Table 1. Mean SMI value was found to be 2.80 in the fully dentate group, 2.99 in the edentu-
Table 1. Descriptive statistics of bone trabeculae parameters

\begin{tabular}{lcccc}
\hline Parameters & Number & Minimum & Maximum & Mean \pm SD \\
\hline SMI & 100 & 0.80 & 4.73 & $2.83 \pm 0.72$ \\
BV/TV & 100 & 0 & 0.96 & $0.29 \pm 0.20$ \\
EF & 100 & -0.51 & 0.72 & $0.4 \pm 0.18$ \\
Tb.Th & 100 & 0.46 & 5.16 & $1.11 \pm 0.61$ \\
Tb.Sp & 100 & 0.62 & 5.76 & $1.99 \pm 1.15$ \\
\hline
\end{tabular}

$\mathrm{SMI}$ - structure model index; BV/TV — bone volume fraction; EF — ellipsoid factor; $\mathrm{Tb}$.Th — trabecular thickness; Tb.Sp — trabecular seperation; SD — standard deviation

lous group, and 2.69 in the unilateral edentulous group. In other words, the trabecular microstructure of the condyle was more rod-shaped in each group. The highest BV/TV value was measured in the fully dentate group whereas the highest EF and Tb.Th values were in the fully dentate group and unilateral edentulous group, respectively, and the highest Tb.Sp value was in the edentulous group. In the microstructure analysis of mandibular condyle of each group, no statistically significant difference was observed between the groups in terms of mean SMI, $\mathrm{BV} / \mathrm{TV}, E F$, and Tb.Th values $(p=0.243, p=0.095$, $p=0.962, p=0.095$, respectively). However, there was significant difference in terms of mean Tb.Sp between the groups; the mean Tb.Sp of edentulous group was significantly higher than the mean of fully dentate group's ( $p=0.021$; Table 2$)$. When the correlation between age and microstructure values were examined, no correlation was observed between age and SMI $(r=-0.062, p=0.540)$, BV/TV $(r=0.037$, $p=0.715)$, Tb.Th $(r=-0.014, p=0.889)$, Tb.Sp 
Table 2. Comparison of trabecular microstructure parameters of edentulous, unilateral edentulous and fully dentate patients using one-way ANOVA (mean \pm SD)

\begin{tabular}{lccccc}
\hline Group & SMI & BV/TV & EF & Tb.Th & Th.Sp \\
\hline Fully dentate & $2.80 \pm 0.67$ & $0.32 \pm 0.16$ & $0.05 \pm 0.17$ & $0.93 \pm 0.25$ & $1.56 \pm 1.14$ \\
Edentulous & $3 \pm 0.70$ & $0.23 \pm 0.20$ & $0.04 \pm 0.16$ & $1.16 \pm 0.53$ & $2.31 \pm 1.05$ \\
Unilateral edentulous & $2.70 \pm 0.79$ & $0.31 \pm 0.22$ & $0.04 \pm 0.20$ & $1.24 \pm 0.85$ & $2.11 \pm 1.16$ \\
$\mathrm{P}$ & 0.243 & 0.095 & 0.962 & 0.095 & 0.021 \\
\hline
\end{tabular}

SMI — structure model index; BV/TV — bone volume fraction; EF — ellipsoid factor; Tb.Th — trabecular thickness; Tb.Sp — trabecular separation; SD — standard deviation

$(r=0.008, p=0.935)$ and $E F(r=-0.038, p=0.707)$ values. In the comparison of SMI, BV/TV, EF, Tb.Th and Tb.Sp parameters of two sides in the unilateral edentulous group, there was no significant difference between two sides in terms of relevant parameters $(p=0.708, p=0.518, p=0.760, p=0.454, p=0.160$, respectively). Good intra-rater reliability was observed in repeated measurements of SMI $(I C C=0.706)$, $\mathrm{BV} / \mathrm{TV}(\mathrm{ICC}=0.774), \mathrm{EF}(\mathrm{ICC}=0.747)$ and $\mathrm{Tb} . \mathrm{Sp}$ $(I C C=0.859)$ in terms of intra-rater reliability values, whereas excellent intra-rater reliability was observed in Tb.Th $($ ICC $=0.986)$.

\section{DISCUSSION}

The results of our study have shown that there was no significant difference between the groups in terms of condyle microstructures excluding Tb.Sp. MRI is the gold standard for demonstrating cartilage structures in TMJ, position of the joint disc, and pathologies such as effusion. However, it is inadequate in the analysis of changes in condyle trabecula due to its relatively low resolution [2]. Methods such as micro-CT, histomorphometric measurements and high-resolution peripheral quantitative CT have been used to evaluate the trabecular structure of the condyle. However, sacrificed dead animal tissues are generally used for these methods $[15,28]$.

In a study by Parsa et al. [24], mandibular images of 20 dry edentulous patients were obtained through $\mathrm{CT}$, micro- $\mathrm{CT}$, and $\mathrm{CBCT}$ and a strong correlation was observed between CBCT and CT $(r=0.89)$ and between $\mathrm{CBCT}$ and micro-CT $(r=0.82)$ when the trabecular microstructure correlations were examined. Therefore, we preferred to use CBCT to obtain images in vivo, it has relatively lower radiation dose, is cost-effective, and has lower acquisition time.

In orthodontic patients with various malocclusion, malocclusion has been shown to increase the prevalence of TMJ disorder. For instance, more than
$60 \%$ of the MRI images of the patients with anterior open bite showed pathological changes in the bone [27]. In patients with malocclusion, canine guidance and posterior occlusion are not ideal. It has been reported that condyles can make unusual deviations to compensate for these imbalances and these unusual movements may cause damage to the joint structures [10]. Furthermore, in a study including about 300 patients, the proportion of open bite patients with and without TMJ disorder complaints was reported to be the same with the abovementioned ratio (about $6 \%$ ) and malocclusion was reported to be not associated with TMJ disorder [4]. Similarly, de Sousa et al. [7] reported in their study of 100 patients that there was no significant difference in joint disorder between patients with and without an anterior open bite, posterior crossbite and more than five posterior teeth lost. Furthermore, in unilateral edentulous cases, the masticatory muscles on both the dentulous and edentulous sides must work harmonically and there is a decrease in the force load on the muscles on both sides. This explains why the resorption process on both sides continues in parallel with each other [6]. Similarly, the condyle trabecular structures on both sides of our patients in the unilateral edentulous group were similarly affected by teeth loss and no significant difference was observed in terms of the microstructure parameters.

Structural model index refers to the average shape of the trabecular structure of the bone. An SMI of 0 is referred to as plate-shaped trabeculae, SMI of 3 is referred to as rod-shaped trabeculae, and SMI of 4 is referred to as sphere-shaped trabeculae [25]. Doube [9] reported that there were concave curvatures to a large extent in the real bone, it was not possible to accurately measure this bone trabecular structure with $\mathrm{SMI}$, and using $\mathrm{EF}$, instead of $\mathrm{SMI}$, in the analysis of trabecular structure can provide more accurate results. They have further reported that $\mathrm{EF}$ gets values 
between -1 and +1 . It gets -1 when discus-shaped ellipsoids are the majority, it gets 0 when there are discus- and javelin-shaped ellipsoids, and it gets +1 when javelin-shaped ellipsoids are the majority. Although there was no significant difference between the three groups in terms of SMI in the present study, the highest mean SMI value was found to be 3 in the edentulous patient group and the lowest mean SMI value was 2.70 in the unilateral edentulous patient group, meaning that the trabecular structure in all three groups was more rod-shaped. When the mean values in all three groups were analysed according to the $\mathrm{EF}$, the trabecular structure was found to be more javelin-shaped. In other words, we can state that SMI and EF parameters reflect trabecular structure similarly.

In a study by Giesen et al. [12] comparing the condyles of the fully dentate and edentulous patients, the edentulous patient group was found to have lower BV/TV values and their bone trabecula was seen to be not as stiff and strong as in fully dentate patients. This reduction in BV/TV was reported to be associated with the conversion of the trabecula to a more rod-like structure, but not associated with the reduction in Tb.Th. In their study, it was not known when edentulous patients lost their teeth or whether they were using dentures. Similarly, in the present study, it was not known how long the edentulous patients had no teeth or whether they were using dentures. No difference was observed between bone microstructure values due to teeth loss in our study. As the BV/TV, i.e. the strength of the bone structure decreased, the trabecula was observed to turn into a more rod-like structure. For instance, the mean $\mathrm{BV} / \mathrm{TV}$ values of the fully dentate and edentulous group were 0.32 and 0.23 , respectively, whereas the mean SMI values were 2.80 and 3 , and Tb.Th values were 0.93 and 1.16 , respectively. As can be seen, the bone trabecular volume in the edentulous group was lower, and its structure turned into a more rod-like pattern. However, the decrease in BV/TV was seen to be not parallel to the decrease in Tb.Th. Findings obtained in our study are compatible with the findings reported by Giesen et al. [12] in their study.

Ding and Hvid [8] analysed the age-related changes in the tibial bone trabecular microstructure values of 40 patients aged 16-85. They reported that the trabecular structure became more rod-like with the increasing age, however, it was further reported that there was no significant change in Tb.Th in patients in the age group of 20-80 and no difference was observed in microstructural values due to the low time-dependent remodelling activity of the tibial bone. In the present study, the duration of teeth loss may not have been long enough to cause remodelling in the trabecular structure of the condyles of the edentulous patients and to affect microstructural values.

\section{Limitations of the study}

This study has some limitations. Firstly, it was not known how long the patients have been edentulous and whether they were using dentures. Secondly, we only used the images of patients participating from the Eastern Anatolia Region of Turkey and the results of our study are, therefore, shaped according to the characteristics of the ethnic structure in this region. In our study, CBCT images with a high resolution of $200 \mu \mathrm{m}$ were used. Since the value range of Tb.Th and Tb.Sp dimensions vary between $0.46-5.16 \mathrm{~mm}$ and $0.62-5.76 \mathrm{~mm}$, it can be said that the voxel size used in our study is sufficient to evaluate the related structures. Furthermore, bone microstructure measurements were seen to be performed in vitro in most of the studies $[5,8,11-14,24,25]$ in the literature whereas our study was carried out in vivo thanks to lower acquisition time and radiation dose provided by CBCT than micro-CT.

\section{CONCLUSIONS}

Although there was no significant difference between the fully dentate, edentulous, and unilateral edentulous patient groups in terms of the trabecular microstructure excluding Tb.Sp of condyle, the trabecular structure of the edentulous group was more rod-like and the mean Tb.Sp of edentulous group was significantly higher than the mean of fully dentate group's. As the trabecular structures of the condyle turned into more rod-like structures, BV/TV value reflecting the strength of the trabecular bone was found to decrease. Moreover, no significant change was observed in trabecular bone microstructure parameters with increasing age.

\section{REFERENCES}

1. Al-Saleh MAQ, Alsufyani NA, Saltaji $H$, et al. MRI and CBCT image registration of temporomandibular joint: a systematic review. J Otolaryngol Head Neck Surg. 2016; 45(1): 30, doi: 10.1186/s40463-016-0144-4, indexed in Pubmed: 27164975.

2. Badel T, Marotti M, Savić Pavičin I, et al. Temporomandibular disorders and occlusion. Acta Clin Croat. 2012; 51: 419-424, indexed in Pubmed: 23339268. 
3. Bogin B, Rios L. Rapid morphological change in living humans: implications for modern human origins. Comp Biochem Physiol A Moll Integr Physiol. 2003; 136(1): 71-84, doi: 10.1016/s1095-6433(02)00294-5.

4. Chiappe G, Fantoni F, Landi N, et al. Clinical value of 12 occlusal features for the prediction of disc displacement with reduction (RDC/TMD Axis I group Ila). J Oral Rehabil. 2009; 36(5): 322-329, doi: 10.1111/j.1365-2842.2009.01942.x, indexed in Pubmed: 19382297.

5. Choi DY, Sun KH, Won SY, et al. Trabecular bone ratio of the mandibular condyle according to the presence of teeth: a micro-CT study. Surg Radiol Anat. 2012; 34(6): 519-526, doi: 10.1007/s00276-012-0943-x, indexed in Pubmed: 22354526.

6. Chou HY, Satpute D, Müftü A, et al. Influence of mastication and edentulism on mandibular bone density. Comput Methods Biomech Biomed Engin. 2015; 18(3): 269-281, doi: 10.1080/10255842.2013.792916, indexed in Pubmed: 23682930.

7. de Sousa ST, de Mello VV, Magalhães BG, et al. The role of occlusal factors on the occurrence of temporomandibular disorders. Cranio. 2015; 33(3): 211-216, doi: 10.1179/2151090314Y.0000000015, indexed in Pubmed: 25027731.

8. Ding M, Hvid I. Quantification of age-related changes in the structure model type and trabecular thickness of human tibial cancellous bone. Bone. 2000; 26(3): 291-295, doi: 10.1016/s8756-3282(99)00281-1.

9. Doube M. The ellipsoid factor for quantification of rods, plates, and intermediate forms in 3D geometries. Front Endocrinol. 2015; 6: 15, doi: 10.3389/fendo.2015.00015, indexed in Pubmed: 25762979.

10. Garcia-Fajardo PC, Casado AC, Trigo AF, et al. La oclusión como factor etiopatológico en los trastornos temporomandibulares. RCOE. 2007; 12(1-2), doi: 10.4321/s1138$123 \times 2007000100003$.

11. Giesen EBW, Ding M, Dalstra $M$, et al. Architectural measures of the cancellous bone of the mandibular condyle identified by principal components analysis. Calcif Tissue Int. 2003; 73(3): 225-231, doi: 10.1007/s00223-002-0002-4, indexed in Pubmed: 14667134.

12. Giesen EBW, Ding M, Dalstra $M$, et al. Changed morphology and mechanical properties of cancellous bone in the mandibular condyles of edentate people. J Dent Res. 2004; 83(3): 255-259, doi: 10.1177/154405910408300314, indexed in Pubmed: 14981130.

13. Giesen E, Ding M, Dalstra M, et al. Reduced mechanical load decreases the density, stiffness, and strength of cancellous bone of the mandibular condyle. Clinical Biomech. 2003; 18(4): 358-363, doi: 10.1016/s02680033(03)00018-4.

14. Hongo $T$, Yotsuya $H$, Shibuya $K$, et al. Quantitative and morphological studies on the trabecular bones in the condyloid processes of the Japanese mandibles. Comparisons between dentulous and edentulous specimens. Bull Tokyo Dent Coll. 1989; 30(2): 67-76, indexed in Pubmed: 2635090.

15. Ibrahim N, Parsa A, Hassan B, et al. Diagnostic imaging of trabecular bone microstructure for oral implants: a literature review. Dentomaxillofac Radiol. 2013; 42(3): 20120075, doi: 10.1259/dmfr.20120075, indexed in Pubmed: 23420864 .
16. Koç A, Eroğlu CN, Bilgili E. Assessment of prevalence and volumetric estimation of possible Stafne bone concavities on cone beam computed tomography images. Oral Radiol. 2020; 36(3): 254-260, doi: 10.1007/s11282-019-00402-4, indexed in Pubmed: 31327093.

17. Koç C, Sönmez G, Yılmaz F, et al. Comparison of the accuracy of periapical radiography with $\mathrm{CBCT}$ taken at 3 different voxel sizes in detecting simulated endodontic complications: an ex vivo study. Dentomaxillofac Radiol. 2018; 47(4): 20170399, doi: 10.1259/dmfr.20170399, indexed in Pubmed: 29376737.

18. Liang X, Liu S, Qu X, et al. Evaluation of trabecular structure changes in osteoarthritis of the temporomandibular joint with cone beam computed tomography imaging. Oral Surg Oral Med Oral Pathol Oral Radiol. 2017; 124(3): 315-322, doi: 10.1016/j.00oo.2017.05.514, indexed in Pubmed: 28732700.

19. Liu ZJ, Yamagata K, Kasahara Y, et al. Electromyographic examination of jaw muscles in relation to symptoms and occlusion of patients with temporomandibular joint disorders. J Oral Rehabil. 1999; 26(1): 33-47, doi: 10.1046/j.13652842.1999.00356.x, indexed in Pubmed: 10080323.

20. Manfredini D, Castroflorio T, Perinetti G, et al. Dental occlusion, body posture and temporomandibular disorders: where we are now and where we are heading for. J Oral Rehabil. 2012; 39(6): 463-471, doi: 10.1111/j.13652842.2012.02291.x, indexed in Pubmed: 22435603.

21. Merrot $O$, Vacher $C$, Merrot $S$, et al. Changes in the edentate mandible in the elderly. Surg Radiol Anat. 2005; 27(4): 265-270, doi: 10.1007/s00276-005-0323-x, indexed in Pubmed: 16215657.

22. Milam SB. Pathogenesis of degenerative temporomandibular joint arthritides. Odontology. 2005; 93(1): 7-15, doi: 10.1007/ s10266-005-0056-7, indexed in Pubmed: 16170470.

23. Nassif NJ, Al-Salleeh F, Al-Admawi M. The prevalence and treatment needs of symptoms and signs of temporomandibular disorders among young adult males. J Oral Rehabil. 2003; 30(9): 944-950, doi: 10.1046/j.13652842.2003.01143.x, indexed in Pubmed: 12950977.

24. Parsa A, Ibrahim N, Hassan B, et al. Bone quality evaluation at dental implant site using multislice $\mathrm{CT}$, micro-CT, and cone beam CT. Clin Oral Implants Res. 2015; 26(1): e1-e7, doi: 10.1111/clr.12315, indexed in Pubmed: 24325572.

25. Sugisaki M, Agematsu H, Matsunaga S, et al. Three-dimensional analysis of the internal structure of the mandibular condyle in dentulous and edentulous jaws using micro-CT. Cranio. 2009; 27(2): 78-87, doi: 10.1179/crn.2009.013, indexed in Pubmed: 19455918.

26. Wang C, Yin X. Occlusal risk factors associated with temporomandibular disorders in young adults with normal occlusions. Oral Surg Oral Med Oral Pathol Oral Radiol. 2012; 114(4): 419-423, doi: 10.1016/j.00oo.2011.10.039, indexed in Pubmed: 22841427.

27. Wang MQ, Xue F, He JJ, et al. Missing posterior teeth and risk of temporomandibular disorders. J Dent Res. 2009; 88(10): 942-945, doi: 10.1177/0022034509344387, indexed in Pubmed: 19783804.

28. Zhang J, Jiao K, Zhang M, et al. Occlusal effects on longitudinal bone alterations of the temporomandibular joint. J Dent Res. 2013; 92(3): 253-259, doi: 10.1177/0022034512473482, indexed in Pubmed: 23340211. 